\title{
UN ESTUDIO SOBRE LA MEJORA EDUCATIVA A TRAVÉS DE LOS GRUPOS INTERACTIVOS EN UNA COMUNIDAD DE APRENDIZAJE
}

In study about improving educational through the interactive group on a community learning

A un estudo melhorar a educação através do grupo interativa em uma comunidade de aprendizagem

\section{Cristina Mateos-Gutiérrez}

Universidad Pablo de Olavide, Sevilla-España. Departamento de Antropología Social, Psicología Básica y Salud Pública. Correo electrónico: cmatgut@upo.es

\section{Resumen}

Los estudios realizados en torno al aprendizaje han sido numerosos a lo largo de las últimas décadas. Buscando una enseñanza eficaz, son muchas las investigaciones que se llevan a cabo al respecto. Uno de los resultados óptimos fruto de los estudios para la mejora educativa son los grupos interactivos. Éstos se muestran como alternativa pedagógica ante las clases magistrales en las que el profesor es el experto conocedor del contenido que se imparte en las aulas, mientras que el alumno es el aprendiz desprovisto de conocimiento. Frente a ello se erige un conocimiento compartido por medio de un aprendizaje dialógico y colaborativo que ofrece el espacio creado por los grupos interactivos. Esta metodología de trabajo dentro del aula es característica de las Comunidades de Aprendizaje sin ser exclusiva de ellas, extrapolándose a cualquier institución escolar. No obstante los rasgos específicos de las Comunidades de Aprendizaje ocasiona la relación indisoluble entre éstas y los grupos interactivos. El estudio que aquí se presenta versa sobre el análisis realizado en grupos interactivos a través de un trabajo etnográfico en una Comunidad de Aprendizaje situada en una zona de exclusión social.

Palabras claves: grupos Interactivos; aprendizaje significativo; aprendizaje dialógico; aprendizaje colaborativo; comunidad de aprendizaje 


\begin{abstract}
Studies about learning have been numerous in the past decades. Seeking to effective teaching, many research are carried out on that subject. One of the best results of studies for educational improvement are interactive groups. These are a pedagogical alternative to the master classes where the teacher is the expert knowledge of the content that is given in the classroom, while the students are devoid of knowledge. Against all this, the best option is a knowledge shared through a dialogic and collaborative learning that the space created by the interaction group stands knowledge offers. This methodology of work in the classroom is characteristic of Learning Communities but is not exclusive of it, this methodology can be extrapolated to other school institution. However, the specific features of the Learning Communities cause the indissoluble link between them and the interactive groups. The study presented here deals with the analysis in interactive groups through an ethnographic work in a learning community located in an area social exclusion.
\end{abstract}

Keywords: interactive groups; meaningful learning: dialogic learning; collaborative learning; learning community

\title{
Resumo
}

Estudos em torno de aprendizagem têm sido numerosos ao longo das últimas décadas. Buscando um ensino eficaz, muitos investigação levada a cabo a este respeito. Um dos resultados bem-sucedida de estudos para melhoria educacional são grupos interativos. Estes são apresentados como uma alternativa educacional às master classes, onde o professor é o conhecimento especializado do conteúdo que é ensinado na sada de aula, enquanto o estudiante está desprovido de apendiz conhecimento. Contra esta compartilhda através de uma aprendizagem dialògica e colaborativa que ofrece o espaco criado pela interação cgrupo está cnhecimento. Esta metodologia de trabalho na sala de aula é característica de Comunidades de Aprendizagem sem ser exclusivo dela, extrapolando qualquer instituição escolar. No entanto, as característicacs específicas das Comunidades de Aprendizagem faz com que a ligação indissolúvel entre eles e os grupos interativos. $\mathrm{O}$ estudo aqui apresentado trata da análise em grpos interativos através de um trabalho etnográfico em uma Comunidade de Aprendizagem localizada em uma área de exclusão social. 
Palavras-chave: grupos interativos; aprendizagem significativa; aprendizagem dialógica; aprendizagem cooperativa; comunidade de aprendizagem

\section{Introducción}

Los grupos interactivos son una metodología de trabajo dentro del aula escolar que facilita los procesos de enseñanza-aprendizaje, promoviendo en el alumnado una mayor autonomía y facilitando la interacción con su grupo de iguales, con el profesorado y con otros participantes del proceso educativo. Su configuración debe ser heterogénea en género, nivel de aprendizaje y grupo de pertenencia del alumnado (Luna, 2007). Son característicos de una Comunidad de Aprendizaje en tanto que ésta se basa en programas desarrollados por medio de la interacción conjunta de todos los integrantes (Elboj et al. 2002). Las Comunidades de Aprendizaje abren la escuela al contexto en el que se encuentran, y posibilitan la participación de toda la vecindad en un plano de igualdad. La participación es la base fundamental de los grupos interactivos, por ello son representativos de las Comunidades de Aprendizaje, pero no exclusivos de éstas. Al igual ocurre con las Comunidades de Aprendizaje que, si bien es cierto que surgieron como mejora del rendimiento académico de escolares que presentaban fracaso escolar, en la actualidad las Comunidades de Aprendizaje se hallan en muy diversos contextos, independientemente de que el entorno responda a una situación más o menos aventajada en relación con la sociedad. Esto es debido al grado de éxito escolar que promueven por medio de la metodología de trabajo que realizan. Esta metodología se basa en la participación comunitaria, en las aportaciones de las familias y vecinos del barrio, en el aprendizaje significativo partiendo de los intereses de los escolares y sus necesidades, en el trabajo colaborativo realizado por medio de los grupos interactivos, alejándose de las enseñanzas tradicionales en las que se considera al maestro poseedor del conocimiento y los niños se muestran pasivos en la participación de las clases.

\section{Marco Teórico}

A partir de la preocupación por un cambio educativo que facilitase el proceso de enseñanza-aprendizaje de escolares que presentaban dificultades de aprendizaje, se llevaron a cabo numerosos estudios para la mejora educativa. Esos estudios van a dar lugar a las Comunidades de Aprendizaje, en donde se ponen en marcha los grupos 
interactivos, pese a que éstos también se realicen en instituciones escolares ordinarias. Entre las investigaciones para la mejora académica de estudiantes cuyos logros no cumplen las expectativas curriculares, destacan los estudios de Michael Cole (Cole, 1999) realizados por el Laboratorio de Cognición Humana y Comparada (LCHC). A través de la intervención en una escuela con niños afroamericanos, Cole y su grupo trabaja sobre los procesos de socialización y escolarización, y la relación existente con los procesos cognitivos de aprendizaje. Otras experiencias similares son las de Olga Vázquez con la 'Clase Mágica' (Vázquez, 2002) o José Luis Lalueza con la 'Casa de Shere Rom’ (Luque y Lalueza, 2013). Junto a ello, se han desarrollado otros programas de Comunidades de Aprendizaje tales como 'School Development Program’ de James Comer (Comer, 1993), 'Accelerated Schools’ en 1986 de Henry Levin (Levin, 1987, 1993) y 'Success for All' en 1987 de Bob Slavin (Slavin, 1997). En España las Comunidades de Aprendizaje surgen a partir de los estudios realizados por el Centro de Investigación en Teorías y Prácticas Superadoras de Desigualdades de la Universidad de Barcelona con la creación del Centro de Educación de Personas Adultas `La VernedaSant Martí en 1978 (Cuenca, 1998). En la actualidad están reguladas por la orden 8 de Junio de 2012.

Conocer los orígenes de las Comunidades de Aprendizaje es relevante en tanto que surgen como interés para un progreso educativo de escolares en situación de exclusión social al no pertenecer al grupo hegemónico desde el que se imparten las enseñanzas escolares, siendo la constitución de dichas comunidades el pilar sobre el que se asientan los grupos interactivos. Éstos, permiten la participación de todos los integrantes del proceso de enseñanza-aprendizaje, haciéndose viable dentro de las enseñanzas institucionalizadas por medio de las Comunidades de Aprendizaje. La participación de la comunidad en la escuela supone un ámbito de socialización en el que la persona es agente activo. Esta participación activa se realiza por medio de la interacción que facilitan los grupos interactivos. A través de éstos se expanden las enseñanzas a toda la vecindad en un proceso de igualdad comunicativa en la que todos son expertos y aprendices. Se aleja la idea de la antigua escuela en la que el profesor era instructor del saber mientras que se consideraban a los alumnos meros aprendices carentes de conocimiento, para abrir camino a un aprendizaje que se construye conjuntamente por medio del trabajo colaborativo y del diálogo igualitario (Elboj et al. 
2002). Desde las Comunidades de Aprendizaje se tiene presente que los niños tienen un saber que se corresponde con el nicho evolutivo, la cultura y los procesos de socialización. De acuerdo a estas tres variables cada escolar tiene una fuente de conocimiento. El nicho evolutivo es el que provee al niño de las normas que son adecuadas en el contexto familiar en el que se desarrolla. La cultura se configura en base a una serie de reglas que son instauradas por los miembros que conforman dicha cultura, y esas reglas se adoptan porque son las que faciliten a los grupos culturales su existencia como grupo, de manera que difieren de las de otros grupos ya que las metas que cada cultura persigue son distintas. A través de los procesos de socialización se va adquiriendo rasgos culturales. Ogbu (1994) realiza una diferenciación entre las diferencias culturales primarias y las secundarias. Las primarias son las que los grupos culturales no pertenecientes a la cultura hegemónica adquieren por elección propia para integrarse en la sociedad, sin que suponga una pérdida de sus características culturales. Las secundarias sin embargo son adquiridas por oposición al grupo hegemónico y en rechazo a éste, por lo que al ser contrarias la integración se dificulta. Si se produce este último caso el grupo cultural se encuentra en una situación de desventaja social ya que desconoce las normas imperantes en la sociedad hegemónica. Si esto sucede, el grupo cultural distinto al resto se ve expuesto a ser dominado, ya que, si uno no domina el sistema, el sistema le domina a él. (Lalueza et al., 2001).

En el caso de la escuela, ésta es un agente socializador importante para los grupos hegemónicos, ya que se corresponden con los modos de vida de dichos grupos. La escuela tiene significado en tanto que se prepara al niño para desarrollar en la edad adulta un trabajo cualificado. En el caso de otros grupos culturales en los que las metas son diferentes y persiguen otros propósitos, la escuela carece de sentido, máxime si los contenidos se imparten desde los cánones de la cultura dominante, de ahí la importancia de las Comunidades de Aprendizaje en las que se promueve un diálogo igualitario y las enseñanzas se imparten por medio de grupos interactivos donde el saber se construye en conjunto a través del aprendizaje colaborativo.

El aprendizaje colaborativo se manifiesta en la medida en que se fomenta la participación guiada por un mediador que es el que procura que el diálogo se genere de forma positiva, permitiendo la intervención a todos los miembros del grupo. Para que el aprendizaje sea colaborativo es necesario la figura de un interventor, la simple creación Un estudio sobre la mejora educativa a través de los grupos interactivos en una comunidad de aprendizaje 
de grupos en los que se facilite la interacción entre los participantes no equivale a que se produzca una colaboración mutua. La figura mediadora dentro de los grupos interactivos es la que promoverá la colaboración y el diálogo igualitario (Moreno, 2016).

El diálogo igualitario permite que se genere una comunicación por la que se comparten conocimientos, en un proceso de construcción de significados compartidos que hace que las explicaciones curriculares se debatan para lograr la comprensión de los contenidos que desde el aula se imparten. Ello promueve la mejora académica y favorece un menor fracaso escolar en los grupos no pertenecientes a la cultura escolar al superar la barrera de la desvinculación originada con los significados que se manejan en uno y otro contexto, el escolar y el del grupo de pertenencia (Rogoff, 1993).

En tanto que se produce la construcción conjunta de significados a través del diálogo igualitario en el aula, se origina un aprendizaje dialógico. El aprendizaje dialógico conlleva a que el profesorado articule un discurso en el que se negocien los significados. Se trata de “...un proceso de construcción social del conocimiento y de cambio conceptual mediante un proceso de intersubjetividad, confrontación y reflexión colaborativa sobre la práctica" (De Vargas, 2006:3). Sobre estos planteamientos se asienta el aprendizaje significativo, término acuñado por Ausubel que se define como "el proceso a través del cual una misma información se relaciona, de manera no arbitraria y sustantiva (no-literal), con un aspecto relevante de la estructura cognitiva del individuo" (Moreira 2000:10).

El aprendizaje significativo permite al alumnado asimilar el conocimiento en base a sus conocimientos previos, alejándose del aprendizaje memorístico característico de las enseñanzas tradicionales. De acuerdo a Ausubel (1963) el aprendizaje memorístico lleva al alumnado a realizar poco o nulo esfuerzo para integrar el nuevo conocimiento que se le presenta con sus conocimientos previos. Dicho autor distingue entre el aprendizaje memorístico, y aquel que dirige al alumno a la interconexión de conocimientos, relacionando el nuevo saber con el saber que posee derivado de su propio proceso socializador -aprendizaje significativo-. En el aprendizaje memorístico el compromiso es escaso, mientras que en el aprendizaje significativo se produce una mayor implicación del alumnado al vincular los contenidos con conceptos que le 
resultan relevantes (Novak, 2011). Esto otorga mayor autonomía al alumno en el proceso de enseñanza-aprendizaje, sin que ello suponga un aprendizaje autodidáctico. El profesor guía y orienta dicho proceso a través del andamiaje.

El andamiaje, concepto desarrollado por David Wood y Jerome Bruner (Bruner, 1969) a partir del postulado de Vygotsky sobre la 'Zona de Desarrollo Próximo' (Vygotsky, 1979, 1995) hace referencia a la ayuda pedagógica que se le ofrece al alumno partiendo de su Zona de Desarrollo Próximo. Éstaqueda definida por Vygotsky como la distancia entre lo que el aprendiz puede realizar por sí sólo 'Zona de Desarrollo Potencial', y las habilidades y destrezas que aún no ha adquirido, para lo cual necesita una ayuda. En este sentido, el andamiaje conlleva inevitablemente la interacción profesor-alumno. En ese proceso de interacción se origina la construcción de significados compartidos por medio del diálogo -aprendizaje dialógico-, bases sobre las que se fundamenta el aprendizaje significativo, cuyos elementos claves son dos: la no arbitrariedad y la sustantividad (Moreira, 2011). La primera hace referencia a la vinculación del contenido con aspectos relevantes para el alumnado, mientras que la segunda se refiere a las diversas formas de expresar un mismo concepto. Ambos elementos aluden a los procesos socializadores del alumnado conforme a los cuales se originan las diversas formas de aprendizaje. Los procesos de socialización de la persona, así como las interacciones que ésta establezca con su medio inciden en los distintos modos de aprender. De acuerdo a lo señalado por Tulviste (1992), no sólo los procesos mentales simples, sino los procesos mentales superiores quedarán determinados por los procesos de socialización, por ello es importante tener presente un aprendizaje significativo que es potenciado a través de los grupos interactivos cuyos fundamentos son el aprendizaje dialógico y el aprendizaje colaborativo.

\section{Objetivo}

Mostrar la mejora educativa de escolares de 6 y 8 años de edad, a través de un aprendizaje basado en grupos interactivos, dentro de un colegio transformado en Comunidad de Aprendizaje situado en una zona de exclusión social.

\section{Metodología}

Se ha lleva a cabo una metodología de corte cualitativo, basada en la etnografía por medio de la observación participante acompañada de un diario de campo realizado al Un estudio sobre la mejora educativa a través de los grupos interactivos en una comunidad de aprendizaje 
finalizar la jornada escolar. La observación participante y el diario de campo se han complementado con entrevistas informales a los docentes del centro y con entrevistas formales a las tutoras de los escolares sobre los que versa el estudio.

El estudio se ha realizado en una Comunidad de Aprendizaje que acoge a población mayoritariamente gitana durante el curso 2014-2015. Dicho estudio se ha llevado a cabo todo el año académico, tres días por semana cinco horas diarias en un curso de segundo de primaria con escolares de edades comprendidas entre los 6 y los 8 años de edad.

El curso de segundo de primaria, dividido en dos grupos de clase, está compuesto por un total de 12 niños en cada grupo. La composición de los grupos es heterogénea tanto a nivel de género como de niveles de aprendizaje. Cada grupo de clase tiene una tutora, éstas trabajan conjuntamente los contenidos que se imparten en ambas clases.

El grupo de clase está dividido en pequeños grupos de trabajo compuesto por 4 niños. Estos grupos de trabajo, son los grupos interactivos. La heterogeneidad en los pequeños grupos se asegura por medio de la rotación periódica de manera que todos los escolares trabajen colectivamente con sus compañeros. Cada grupo tiene un número para identificarlos, y cada niño tiene un número para que la rotación sea plena. La rotación de los grupos numerados es realizada periódicamente. El cambio de niños de un grupo a otro se realiza en función de las demandas del grupo. Si un niño necesita más apoyo y tiene más afinidad con un compañero que le pueda ofrecer ese apoyo, se realiza el cambio de niños dentro del grupo. En cada grupo interactivo hay una persona adulta que guía el proceso junto con la tutora del grupo. Las personas adultas que están en los grupos interactivos son personal docente del centro, estudiantes universitarios, personal de prácticas o jóvenes voluntarios.

Todos los grupos realizan la misma actividad, sólo que la llevan a cabo en diferentes momentos. Los grupos al estar numerados, realizan actividades distintas hasta que todos han hecho todas las actividades. De esta manera el grupo 1 comienza con una actividad de lectoescritura, el grupo 2 con una actividad de matemáticas, el grupo 3 con una actividad de conocimiento del medio, y el grupo 4 con una actividad de ciencias sociales. El tiempo es limitado para que cada grupo realice la actividad. Si un niño no la 
ha terminado, deja la ficha inacabada en su casillero para terminarla en momentos en los que haya finalizado con mayor rapidez otra actividad, o en la materia de plástica cuando la han terminado o prefieren cambiar de actividad y finalizar la ficha. Se muestra aquí cómo el interés del niño incita a su motivación. Una vez que el tiempo para realizar la actividad ha finalizado, los grupos de niños rotan hacia otras mesas donde está el adulto. Los adultos que guían las actividades no rotan. La duración estimada para que los cuatro grupos hayan realizado las cuatro actividades es aproximadamente de 45 minutos. Una vez finalizado ese tiempo todos los niños se sientan en círculo junto con los demás adultos y tutora para comentar las complejidades que se han encontrado, aquellos contenidos que les han resultado más interesantes o aquellos que les han sido más fáciles realizar.

Por medio de esta metodología de trabajo, los niños aprenden a trabajar en equipo, ya que en los grupos interactivos los escolares se ayudan unos a otros, mientras que la persona adulta guía el proceso. Además, se negocian los significados a través del diálogo que se genera dentro del grupo. Por otro lado, se produce un andamiaje en el que el niño o el adulto, en función de la complejidad de la actividad, guía a otros niños.

Estos datos se han analizado en base a la observación por medio de notas de campos que se han ido tomando en pequeños momentos a lo largo de toda la jornada escolar durante todo el tiempo que se ha estado realizando la etnografía (septiembre 2014 - junio 2015). Posteriormente, cada día una vez finalizada la jornada en el colegio se llevaba a cabo el diario de campo. La inmediatez con la que se realizaba el diario de campo facilitaba que los interrogantes que se planteaban ante la nueva realidad, así como las situaciones que sorprendían, fueron recogidas de la forma más exhaustiva.

Para analizar el diario de campo se realizó un sistema de categorías que engloba: relaciones igualitarias, conflictos, interés por la actividad, resolución de problemas sin andamiaje, resolución de problemas con andamiaje de los iguales, resolución de problemas con andamiaje de un adulto.

Las relaciones igualitarias eran patentes en todo momento ya que tanto niños como adultos estaban sentados en el mismo nivel y tenían la misma posibilidad de realizar aportaciones en relación a la actividad. 
Los conflictos eran comunes cuando los niños desconocían cómo resolver el problema, entonces se distraían o empezaban a jugar entre ellos dándose patadas que terminaban en peleas.

El interés por la actividad está vinculado con los conflictos, ya que, si al niño se le muestra la actividad como motivadora pese a que desconozca cómo resolverla, muestra atención y pide ayuda, no ocasionándose el conflicto.

El andamiaje entre iguales se ha registrado más veces que el andamiaje con adultos. Los niños prefieren ayudarse entre ellos siempre que puedan.

De las entrevistas informales a los docentes del centro en conversaciones esporádicas que surgían de manera espontánea, se han recogido datos referentes al cambio del centro desde que es una Comunidad de Aprendizaje y a la mejora del proceso de enseñanza-aprendizaje de los niños. De acuerdo a lo que verbalizan los docentes, el centro escolar antes de transformarse en Comunidad de Aprendizaje era un colegio de educación infantil y primaria en el que los conflictos entre las familias y la escuela eran constantes. Conflictos traducidos en agresiones físicas y verbales dentro del hall del mismo colegio. Desde que se produjo el cambio a Comunidad de Aprendizaje las agresiones se redujeron hasta desaparecer, debido a que las familias participaron en el proyecto de creación del colegio en Comunidad de Aprendizaje y el recelo que en un inicio les ocasionaba, ya no existía, pues sus aportaciones eran tenidas en cuenta lo que genera un sentimiento de pertenencia dentro de la Comunidad de Aprendizaje,

En relación a los procesos de enseñanza-aprendizaje, los datos obtenidos de las entrevistas informales con los agentes educativos del centro señalan progresos en relación a una disminución del fracaso y el abandono escolar, un incremento de los resultados obtenidos al final del curso, y unas mejores relaciones entre la familia y la escuela que favorece lo anteriormente señalado. La disminución del fracaso escolar y el abandono escolar conllevan inevitablemente una mejora de los resultados, pues los niños con su asistencia a clase y trabajando por medio de grupos interactivos adquieren un mejor conocimiento de los contenidos, involucrándose más en la actividad al ser partícipes activos de la misma. 
De las entrevistas formales realizadas a las tutoras ambas coinciden en la necesidad de que se trabaje de forma heterogénea y en grupo. Destaca el comentario de una maestra cuando hace referencia al aprendizaje en grupos interactivos tan lejano al aprendizaje memorísticos de las enseñanzas tradicionales. "lo hay que enseñarle al niño es a decir 'no', y que tenga su propio criterio”. Explica que los niños deben reflexionar sobre el contenido que se les plantea, sin tener miedo a equivocarse, esto es lo que provoca la participación activa del niño, involucrándose en su propio proceso de enseñanza-aprendizaje, siendo ellos los protagonistas de dicho proceso, lo que origina un menor abandono escolar.

\section{Resultados}

Los resultados extraídos de la observación participante, el diario de campo analizado y de las entrevistas transcritas y analizadas demuestran una mejora educativa de los escolares cuando trabajan en grupos interactivos. Dicha mejora se refleja en la mayor facilidad para asimilar los contenidos curriculares y en la mayor implicación por parte del alumnado.

La mejor comprensión de los contenidos curriculares deviene de dos fuentes: las explicaciones que el alumnado ofrece a su grupo de iguales con un lenguaje que comparten, y la negociación de significados que se origina por parte de los adultos hacia el grupo de escolares cuando detectan que un niño no ha comprendido la materia.

Las explicaciones que dentro del grupo interactivo ofrece un niño a su grupo de iguales, utilizando un lenguaje similar al de sus compañeros, rompe la desvinculación ocasionada por los distintos sistemas de códigos y significados que utiliza la escuela frente a los que utiliza el alumnado. Un ejemplo de ello que se ha observado de forma constante es la espontaneidad del alumnado para hacer comprensible las explicaciones de los adultos. Esto se produce cuando las tutoras han expuesto el trabajo que deben realizar al gran grupo de clase y éste se distribuye en los grupos interactivos. En el momento en el que se comienza a realizar la tarea y un niño no ha comprendido qué o cómo tiene que realizarla, la espontaneidad por parte del alumnado dentro del grupo interactivo para hacer comprensible al compañero la tarea con un lenguaje más cercano, es inminente. Esto se produce gracias al aprendizaje colaborativo. 
Por otro lado, la mejor asimilación de la materia se da a través de la negociación de significados por parte de los adultos cuando detectan que un niño no ha comprendido la explicación. Esto se origina por medio del aprendizaje dialógico. A través de éste se produce la construcción conjunta de significados que lleva a adecuar el lenguaje al sistema de códigos y significados del alumnado. Ello se ha observado en los momentos en los que las tutoras reúnen al grupo de clase en 'Asamblea', espacio configurado en círculo donde todos están sentados al mismo nivel, asemejándose a un gran grupo interactivo en la medida en que por medio de un diálogo igualitario se explican los contenidos que se van a trabajar en los grupos interactivos. Cuando las tutoras detectan que sus explicaciones resultan incomprensibles para algunos niños, hacen uso de ejemplos cotidianos a la realidad del niño para que la comprensión de los contenidos resulte eficaz, creándose la construcción de significados compartidos.

Los grupos interactivos por lo tanto promueven una mejora educativa en tanto que se produce una mejor asimilación de la materia curricular a través de la adecuación del lenguaje utilizado -ya sea por parte de las explicaciones del grupo de iguales o por la negociación de significados- y promueve igualmente una mejora educativa en la medida en que se produce una mayor implicación del alumnado en el proceso educativo.

La mayor implicación del alumnado se refleja en dos aspectos. Uno de los aspectos es el empoderamiento que adquiere el alumnado cuando facilita las explicaciones a sus iguales. Se ha observado un ejemplo de ello cuando los adultos están ofreciendo apoyo a otros niños, y el niño que facilita a su compañero la comprensión de la materia adquiere de forma voluntaria el rol de profesor corrigiendo al compañero la tarea que han realizado.

El otro aspecto que refleja la implicación del alumnado se produce cuando dentro del grupo interactivo se ofrece a los escolares la oportunidad de expresar sus conocimientos más allá de los académicos. En este caso las tutoras incentivan a los niños por medio de preguntas o siendo ellas quienes inician el `saber compartido’. Un ejemplo de ello es la 'Asamblea' nombrada anteriormente, que tiene lugar a primera hora de la mañana. En ella, antes de que se expliquen los contenidos que se van a trabajar, las tutoras cuentan experiencias personales y les preguntan a los niños por las suyas. Esto sitúa al grupo de clase en una condición de igualdad haciéndoles partícipes 
activos del proceso de enseñanza-aprendizaje -entendido éste como proceso de construcción de conocimientos y no mera transmisión de contenidos curriculares-. En la medida en que los escolares tienen la posibilidad de aportar su saber al grupo y es partícipe del mismo, se involucra. Esta implicación se refleja en el hecho de que la mayoría de los niños en la 'Asamblea' levanten la mano para narrar sus experiencias y compartir sus conocimientos.

Se evidencia por lo tanto la mejora educativa en tanto que se produce un empoderamiento de los niños por medio del aprendizaje colaborativo, y un compromiso de éste al dar cabida a sus conocimientos por medio del aprendizaje dialógico.

\section{Discusión}

La metodología curricular por medio de la creación de grupos interactivos, conlleva una mayor receptividad por parte del alumnado ante los contenidos académicos en el momento en el que participa activamente en el proceso de enseñanza-aprendizaje, ya sea a través del aprendizaje colaborativo, o por medio del aprendizaje dialógico. La posibilidad de ofrecer apoyo pedagógico a sus iguales o compartir sus conocimientos con el grupo de clase, hace al alumnado partícipe de las enseñanzas escolares.

La participación de los niños despierta su interés escolar en tanto que se convierten en agentes activos y se adaptan las enseñanzas a su sistema de códigos y significados, superando la barrera generada por la desvinculación del lenguaje escolar y el del alumnado. De acuerdo al proceso socializador del grupo de pertenencia del alumnado, el sistema de códigos y significados variará en relación a la de otros grupos, ya que los significados se construyen en el contexto en el que se socializa la persona (Vygotsky, 1995). En el caso de grupos no pertenecientes a los grupos hegemónicos sobre los que se basan los cánones de la escuela, la construcción conjunta de significados se muestra esencial para una mejora educativa. A través de la colaboración conjunta "se desarrolla una 'ideocultura' común que permite el acceso a significados compartidos; y los participantes colaboran en tareas situadas en la zona de desarrollo próximo" (Luque y Lalueza, 2013:405). El espacio dialogante que ofrece los grupos interactivos permite la construcción conjunta de significados, y para que así sea, la configuración de estos grupos debe ser heterogénea, diversificando género y ritmos de aprendizaje. La heterogeneidad en la configuración del grupo es la que procurará la 
construcción de conocimientos compartidos, dado que las aportaciones que cada niño realice versarán sobre su proceso socializador (Rogoff, 1993).

La construcción de significados compartidos promueve un aprendizaje significativo, lo que facilita la mejor comprensión de los contenidos curriculares favoreciendo la implicación del alumnado al hacer accesible las enseñanzas a su sistema de códigos y significados. Los grupos interactivos permiten además el trabajo colaborativo que lleva al alumnado a adquirir un compromiso escolar en el momento en el que asumen de manera voluntaria el rol de profesor al corregir la tarea de su compañero en los casos en los que las profesoras no puedan atender la inmediatez de sus demandas. Los grupos interactivos por lo tanto promueven una mejora educativa en tanto que se favorece la autonomía del aprendizaje, se comparten conocimientos, y se negocian significados.

\section{Contribuciones}

La mejora educativa que confieren los grupos interactivos al producirse una mayor asimilación de los contenidos curriculares y una mayor implicación en el proceso de enseñanza-aprendizaje, posibilita la reducción del fracaso escolar del alumnado y una mejora del rendimiento académico. El menor fracaso escolar y la mejora académica del alumnado provocan un sentimiento de autoeficacia al ver conseguido los logros académicos que desde las instituciones escolares se estipulan. Dicho sentimiento de autoeficacia da lugar a la autoconfianza y por ende a la mejora de la autoestima (Walsh, 1998), lo que favorece la toma de decisión en un proceso de crecimiento personal del alumno dado que la escuela debe partir de una enseñanza formal contribuyendo a un crecimiento de la persona para que se desenvuelva con éxito en la sociedad ya que la educación formal debe procurar no sólo la adquisición de conocimientos sino el desarrollo del crecimiento personal para que el escolar se desenvuelva con éxito en la sociedad (Coll, 1991).

Debido a que la sociedad en la que vivimos tiene como meta la construcción de la persona para que en la edad adulta se desarrolle con éxito en el mundo laboral, la escuela no sólo se constituye como principal agente socializador, sino como pilar básico para la integración de la persona en la sociedad. La importancia de que las enseñanzas versen sobre contenidos significativos y una metodología de trabajo como la expuesta, 
garantiza que los grupos pertenecientes a otras culturas cuyas metas son distintas a las de la sociedad en la que se encuentran, se introduzcan en el sistema educativo al tener en cuenta sus intereses y partir de la negociación de significados que se generan en los procesos de socialización por medio del diálogo igualitario.

Los grupos interactivos se muestran pues como alternativa a la mejora educativa, prevención del fracaso escolar y el abandono escolar, sobre todo en aquellos escolares que pertenecen a grupos culturales distintos al hegemónico.

\section{Referencias bibliográficas}

Ausubel, D.P (1963). The psychology of meaningful verbal learning. New York: Grune and Stratton.

Bruner, J. S. (1969). Hacia una teoría de la instrucción. México: Uteha.

Cole, M. (1999). Psicología cultural: una disciplina del pasado y del futuro. Madrid: Morata.

Coll, C. (1991). Psicología y curriculum. Barcelona: Paidós.

Comer, J. (1993). School Power: implications of an intervention project. New York: Simon and Schuster.

Cuenca, J.M. (1998). Centro de EPA La Verneda Sant-Martí. Un pequeño milagro cotidiano. Cuadernos de Pedagogía, 265, 33-40.

De Vargas, E. (2006). La situación de enseñanza y aprendizaje como sistema de actividad: el alumno, el espacio de interacción y el profesor. Revista iberoamericana de Educación, 39 (4) 1.

Elboj Saso, C.; Puigdellívol Aguadé, I.; Soler Gallart, M.; Valls Carol, R. (2002). Comunidades de Aprendizaje. Transformar la educación. Barcelona: Graó.

Lalueza, J.L.; Crespo, I.; Pallí, C.; Luque, M.J. (2001). Socialización y cambio cultural en una comunidad étnica comunitaria. El nicho evolutivo gitano. Cultura y Educación 13 (1), 115-130.

Levin, H.M. (1993). The accelerated schools resource guide. San Francisco: JosseyBass Publishers. 
- (1987). New school for the disadvantage. Teacher Education Quarterly. Vol. 14. $\mathrm{N}^{\circ} 4$.

Luna, F. (2007). Aprender en Grupos Interactivos. Cuadernos de Pedagogía, 368, 1823.

Luque, C. y J.L. Lalueza (2013). Aprendizaje colaborativo en Comunidades de práctica en entornos de exclusión social. Revista de Educación, 362, 402-428.

Moreira, M.A. (2011). Aprendizaje significativo: Un concepto subyacente. Instituto de Física: UFRGS.

- (2000). Aprendizaje significativo: teoría y práctica. Madrid: Visor.

Moreno, A. (2016). Grupos Interactivos en la Comunidades de Aprendizaje Mosaico de $\begin{array}{llllll}\text { Santiponce. } & \text { Consulta } & \text { realizada } & 8 & \text { agosto } & 2016\end{array}$ http://www.juntadeandalucia.es/educacion/webportal/web/revista-andaluciaeducativa/en-portada/-/noticia/detalle/grupos-interactivos-en-la-comunidad-deaprendizaje-mosaico-de-santiponce-1

Novak, J.D. (2011). Meaningful learning underlines the constructive integration of thinking, feeling, and acting leading to empowerment for commitment and responsibility. Meaningful Learning Review, 1 (2), 1-14.

Ogbu (1994). From Cultural Differences to Differences in Cultural frame of Refence. En P.M. Greenfield y R.R. Cocking (Eds), Cross-cultural Roots of Minority Child Development (pp. 369-391). Hillsdale, N.J: LEA.

Orden de 8 de Junio de 2012, por la que se regula el procedimiento de inscripción y continuidad de centros reconocidos como "Comunidad de Aprendizaje" y se crea la Red Andaluza “Comunidades de Aprendizaje”. (BOJA no 126 de 28-06-2012) $\begin{array}{lllll}\text { Consulta } & \text { realizada } & 21 & \text { agosto }\end{array}$ http://www.juntadeandalucia.es/boja/2012/126/21

Rogoff, B. (1993). Aprendices del pensamiento. El desarrollo cognitivo en el contexto social. Barcelona: Paidós.

Slavin, R.E. (1997). Educational Psychology. Theory into practice. Nueva Jersey: Prentice Hall. 
Tulviste, P. (1992). Diversidad cultural y heterogeneidad en el pensamiento. Apuntes de Psicología, 35, 5-15

Tudge, J. (1993). Vygotsky, la zona de desarrollo próximo y la colaboración entre pares: connotaciones para la práctica del aula. L. Moll (Comp.), Vygotsky y la educación. Connotaciones y aplicaciones de la Psicología Sociohistórica en la educación (pp. 187-207). Aique: Grupo Editor.

Vázquez, O. (2002). La Clase Mágica: Imagining Optimal Possibilities in a Bilingual Community of Learners. New Jersey: Laurence Erlbaum Publishers.

Vygotsky, L. S. (1995). Pensamiento y lenguaje. Barcelona: Paidós.

- (1979). El desarrollo de los procesos psicológicos superiores. Buenos Aires: Grijalbo.

Walsh, F. (1998). El concepto de resiliencia familiar: crisis y desafío. Buenos Aires: ASIBA. 